\title{
Numerical Simulation of Exogenous Type Microbial Depolymerization Process with Weight Distribution and Microbial Population
}

\author{
Masaji Watanabe, Fusako Kawai
}

\begin{abstract}
Results from previous studies were incorporated into a simulation of a microbial depolymerization process. Experimental results were incorporated into analysis, and a mathematical model for a weight distribution and a microbial population was analyzed numerically. Numerical results and experimental results were compared, and the validity of our model and numerical techniques are discussed.
\end{abstract}

Keywords - biodegradation, polymer, mathematical model, numerical simulation

\section{Introduction}

Molecules liberate monomer units from their terminals in an exogenous type microbial depolymerization process. Polyethylene (PE) and polyethylene glycol (PEG) are polymers that are subject to exogenous type microbial depolymerization processes. Microbial depolymerization processes of PEG have been documented since the last century. Results in those studies include utilization of PEG of average molecular weight 20000 by Pseudomonas aeruginosa [1], degradation of PEG 20000 by anaerobic bacteria isolated from sludge of a municipal anaerobic digester [2], and efficient biodegradation of PEG by Pseudomonas stutzeri [3]. Microbial depolymerization processes of exogenous type have also been studied analytically and numerically. A mathematical model was formulated and numerical techniques were developed in studies of biodegradation of PE [4]. Numerical techniques developed for PE biodegradation were reapplied to microbial depolymerization processes of PEG [5].

Random breakdown of molecules is the main mechanism of endogenous type depolymerization processes. Polyvinyl alcohol (PVA) and polylactic acid (PLA) are polymers subject to endogenous type depolymerization processes. A mathematical model was formulated and numerical techniques were applied to an enzymatic degradation process of PVA [6].

Masaji Watanabe

Okayama University

Japan

Fusako Kawai

Kyoto Institute of Technology

Japan
The numerical techniques originally developed for enzymatic degradation of PVA were reapplied to an enzymatic hydrolysis of polylactic acid (PLA) [7]. Techniques originally developed for endogenous type processes were applied to exogenous type processes of PE and PEG [8].

This study revisited a PEG biodegradation process. Experimental outcomes obtained by cultivation of microbial consortium E-1 in culture media, in which PEG was the only carbon source, were incorporated in a numerical study. Results from a previous study were incorporated in a numerical simulation of an exogenous type depolymerization of PEG.

\section{Description of Model and Results from Previous Studies}

Denote by $w(t, M)$ [mg] the weight distribution of a polymer with respect to the molecular weight $M$ at time $t$. Let $v(t)[\mathrm{mg}]$ be the total weight of polymer molecules with molecular weight between $A$ and $B$ at time $t$. The total weight $v(t)$ is expressed in terms of the integral

$$
v(t)=\int_{A}^{B} w(t, M) d M .
$$

The total weight $v(t)$ of the entire residual polymer or residual polymer at time $t$ is expressed in terms of the integral

$$
v(t)=\int_{0}^{\infty} w(t, M) d M
$$

Integral (1) is an approximation of the integral (2) with appropriate values of $A$ and $B$. In this study, integral (2) was replaced with an integral (1) with $A=10^{3.1}$ and $B=10^{4.2}$. An integral with the lower limit 0 was replaced with an integral with the lower limit $A=10^{3.1}$, and an integral with the upper limit $\infty$ was replaced with an integral with upper limit $B=10^{4.2}$.

Denote by $\sigma(t)$ the total population of viable cells at time $t$. System of equations

$$
\begin{aligned}
& \frac{\partial w}{\partial t}=\sigma(t)\left[-\lambda(M) w+c(M) \int_{M}^{\infty} \lambda(K) d(K) w(t, K) d K\right], \\
& \frac{d \sigma}{d t}=k\left[-v^{\prime}(t)\right]-h \sigma, \\
& c(M)=M e^{\rho M}, d(K)=\frac{\rho e^{-\rho K}}{K\left(1-e^{-\rho K}\right)}, \rho=\frac{\log 2}{L},
\end{aligned}
$$

was proposed in previous studies [10 - 15]. Here, constant $L$ is the molecular weight of a monomer unit, e.g. PE: $L=28$ $\left(\mathrm{CH}_{2} \mathrm{CH}_{2}\right)$, PEG: $L=44\left(\mathrm{CH}_{2} \mathrm{CH}_{2} \mathrm{O}\right)$, and $k$ and $h$ are positive 
parameters. Functions $\lambda(M)$ is the molecular factor of the degradation rate, whereas microbial population $\sigma(t)$ is the time factor of degradation rate. Equations (2) and (3) lead to

$$
v^{\prime}(t)=\sigma(t) \int_{0}^{\infty}\left[-\lambda(M) w(t, M)+c(M) \int_{M}^{\infty} \lambda(K) d(K) w(t, K) d K\right] d M
$$

Denote by $f_{0}(M)$ and $\sigma_{0}$ the initial weight distribution and the initial microbial population, respectively. Equations (3), (4) are associated with the initial conditions

$$
\begin{gathered}
w(0, M)=f_{0}(M), \\
\sigma(0)=\sigma_{0}
\end{gathered}
$$

The initial value problem (3), (4), (6), (7) can be tackled provided the molecular factor $\lambda(M)$ and values of the parameters $\sigma_{0}, k$, and $h$ are prescribed. Techniques for inverse analysis for the function $\lambda(M)$ and the parameters $\sigma_{0}, k$, and $h$, were developed in previous studies. The change of variables from $t$ to $\tau$

$$
\tau=\int_{0}^{t} \sigma(s) d s .
$$

was applied to the equations (3) and (4). Denote the functions corresponding to $w(t, M), \sigma(t)$, and $v(t)$ according to the transformation (8) by $W(\tau, M), S(\tau)$, and $V(\tau)$, respectively. In view of the relation

$$
\frac{\partial}{\partial \tau}=\frac{\partial}{\partial t} \frac{\partial t}{\partial \tau}=\frac{1}{\sigma(t)} \frac{\partial t}{\partial \tau},
$$

equations (3) and (4) are transformed to

$$
\begin{aligned}
& \frac{\partial W}{\partial \tau}=-\lambda(M) W+c(M) \int_{M}^{\infty} \lambda(K) d(K) W(\tau, K) d K, \\
& \frac{d S}{d \tau}=k V^{\prime}(\tau)-h,
\end{aligned}
$$

respectively. In view of the relation

$$
\begin{aligned}
& v^{\prime}(t)=\frac{d v}{d t}=\frac{d V}{d \tau} \frac{d \tau}{d t}=V^{\prime}(\tau) \sigma(t), \\
& V^{\prime}(\tau)=\int_{0}^{\infty}\left[-\lambda(M) W(\tau, M)+c(M) \int_{M}^{\infty} \lambda(K) d(K) W(\tau, K) d K\right] d M .
\end{aligned}
$$

Let $F_{1}(M)$ be the weight distribution for $\tau=T_{1}$, that is,

$$
W\left(T_{1}, M\right)=F_{1}(M)
$$

and let $F_{2}(M)$ be the weight distribution for $\tau=T_{2}$, that is,

$$
W\left(T_{2}, M\right)=F_{2}(M) .
$$

Equation (9), the initial condition (11), and the final condition (12) form an inverse problem for $\lambda(M)$, for which the solution of the initial value problem (9), (11) satisfies the final condition (12). In previous studies, weight distributions of PEG before after cultivation of microbial consortium E-1 for two days, four days, and seven days, $f_{0}(M), f_{1}(M), f_{2}(M)$, and $f_{3}(M)$, were introduced into analysis. Note that $w\left(t_{i}, M\right)=f_{i}(M)$ $(i=0,1,2,3)$ for $t_{0}=0, t_{1}=2, t_{2}=4$, and $t_{3}=7$. Functions $f_{1}(M)$ and $f_{2}(M)$ were assigned to $F_{1}(M)$ and $F_{2}(M)$, respectively, and the inverse problem (9), (11), (12) was solved numerically for $T_{1}=0$ and $T_{2}=2$.
Once the inverse problem for $\lambda(M)$ was solved, equation (9) was solved for $W(\tau, M)$ with the initial condition

$$
W\left(\tau_{0}, M\right)=f_{0}(M),
$$

where $\tau_{0}=0$, A previous study shows that function $V(\tau)$ is approximated with an exponential function

$$
V(\tau)=v_{0} e^{-\mu \tau} \quad\left(v_{0}=\int_{0}^{\infty} f_{0}(M) d M\right)
$$

Note that $V^{\prime}(\tau)=-\mu v_{0} e^{-\mu \tau}$. In a previous study, function $V(\tau)$ was approximated with the exponential function (13) with $\mu \approx 0.506$ [16].

Once $\quad V(\tau) \quad$ was obtained, equations $V\left(\tau_{i}\right)=v\left(t_{i}\right) \quad(i=1,2,3)$ were solved numerically to find values $\tau_{1} \approx 0.634, \tau_{2} \approx 2.580$, and $\tau_{3} \approx 6.863$.

Let $S\left(\tau, \sigma_{0}, k, h\right)$ denote the solution of the equation (11) with initial value $\sigma_{0}$. The change of variables (8) leads to the expression $t=q\left(\tau, \sigma_{0}, k, h\right)$, where

$$
q\left(\tau, \sigma_{0}, k, h\right)=\int_{0}^{\tau} \frac{d r}{S\left(r, \sigma_{0}, k, h\right)} .
$$

Given three pairs of values of $t$ and $\tau$, equations

$$
g_{1}\left(\sigma_{0}, k, h\right)=0, g_{2}\left(\sigma_{0}, k, h\right)=0, g_{3}\left(\sigma_{0}, k, h\right)=0,
$$

where $g_{i}\left(\sigma_{0}, k, h\right)=q\left(\tau_{i}, \sigma_{0}, k, h\right)-t_{i}$, were solved, and values of the parameters $\sigma_{0} \approx 0.127, k \approx 0.00380$, and $h \approx 0.0821$ were obtained [16]. In this study, $\sigma_{0} \approx 0.127, k \approx 0.00379$, and $h \approx 0.0814$ were obtained.

\section{Numerical Simulation Based on Initial Value Problem}

Once the molecular factor of the degradation rate $\lambda(M)$ and the value of the parameters $\sigma_{0}, k$, and $h$ were obtained, initial value problem (3), (4), (6), (7) was solved numerically. Figures 1 - 4 show numerical results. Figures 1 and 2 show numerical results for weight distributions after cultivation of the microbial consortium E-1 for two days, four days, and seven days. The figures also show experimental results for weight distributions before and after cultivation of the microbial consortium E-1. Figure 3 shows the numerical result for the microbial population $\sigma(t)$.

The optical density OD 630 was recorded before and after cultivation of the microbial consortium E-1. Figure 4 shows values of optical density OD 630, $O_{0}, O_{1}, O_{2}$, and $O_{3}$ for $t_{0}$ $=0, t_{1}=2, t_{2}=4$, and $t_{3}=7$, and the OD conversion of the microbial population. 


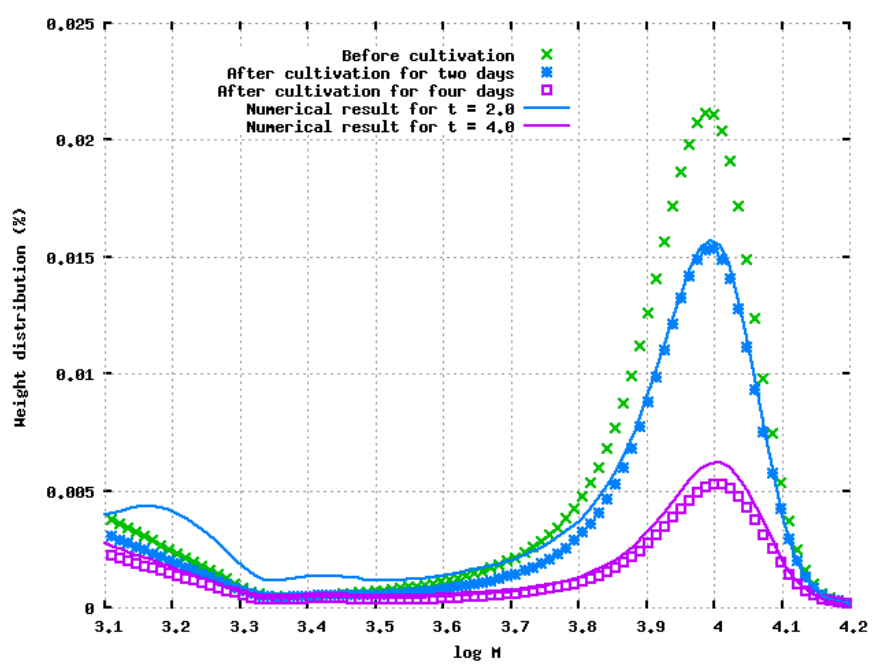

Figure 1: Weight distributions before and after cultivation of microbial consortium E-1 for two days and four days.

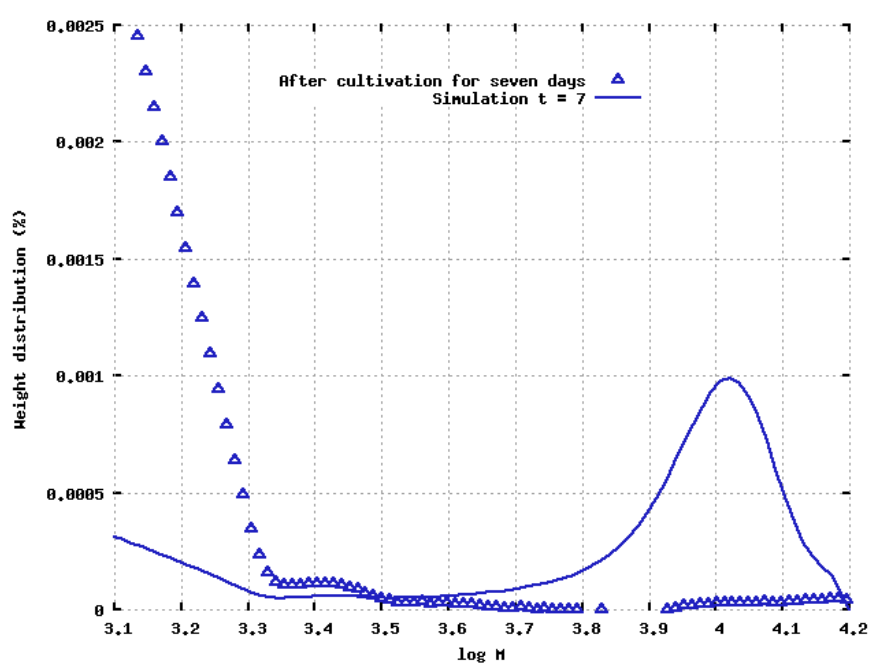

Figure 2: Weight distribution after cultivation of microbial consortium E-1 for seven days.

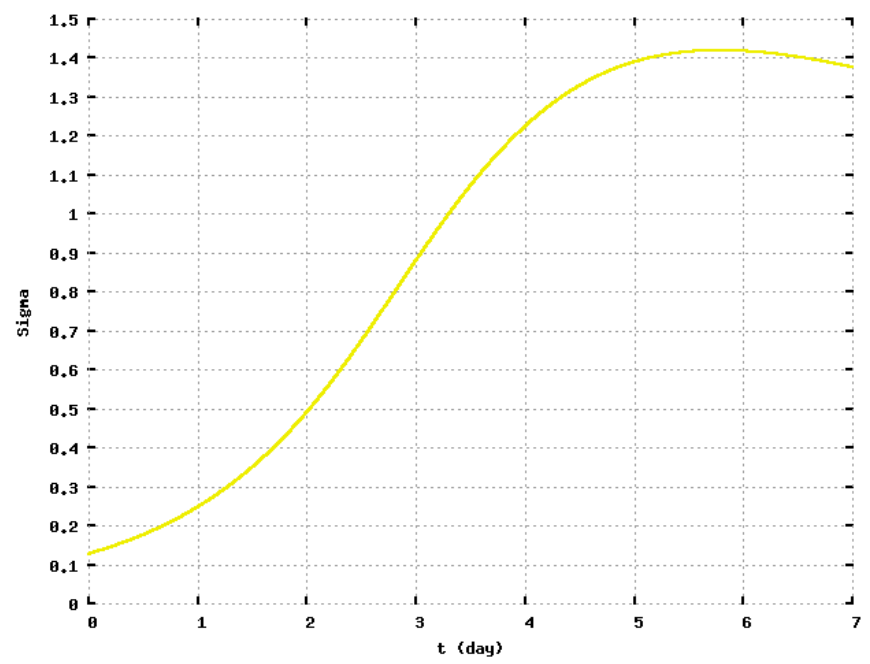

Figure 3: Curve

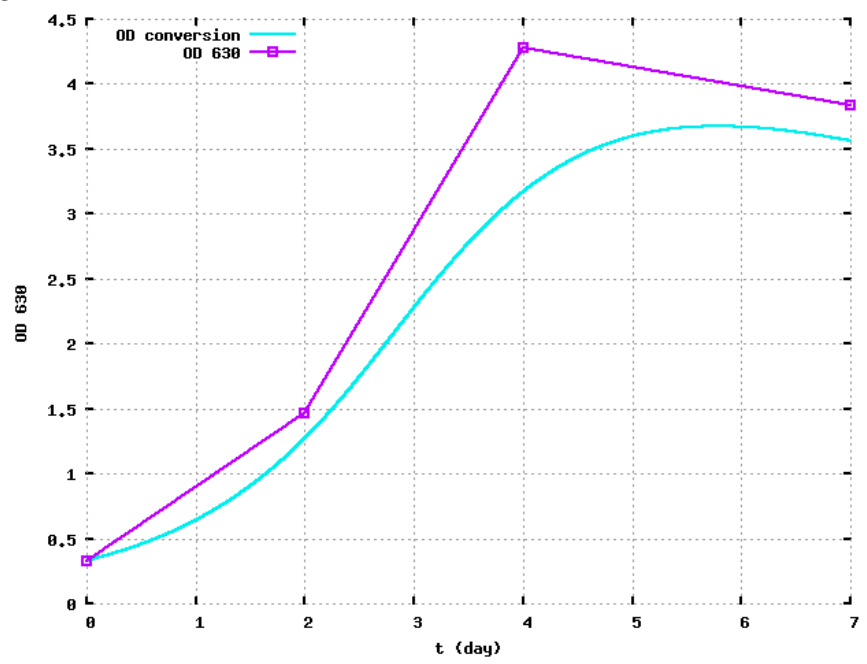

Figure 4: OD 630 and OD conversions of microbial population. OD 630, $O_{0}, O_{1}, O_{2}$, and $O_{3}$ for $t_{0}=0, t_{1}=2, t_{2}$ $=4$, and $t_{3}=7$, and the OD conversion of the microbial population $\left(t, \sigma(t) / \sigma(0) * O_{0}\right) \quad(0 \leq t \leq 7)$ are shown.

\section{Discussion}

In a previous study [16], initial value problem (3), (6) was solved with , after the inverse problem was solved. In this study, initial value problem (3), (4), (6), (7) was solved after the inverse problems for and parameters , $\mathrm{k}$, and $\mathrm{h}$, were solved. Figures 1 and 2 show numerical results and experimental results for the weight distributions after cultivation of the microbial consortium E-1 for two days, four days and seven days. The scales of vertical axes are different, and the discrepancy between the experimental result and the numerical result that Figures 2 shows is not so large as it may appear. Reasonable agreements are shown between the numerical results and the experimental results for weight distributions after cultivation, which shows that our techniques are applicable to simulation of exogenous type microbial depolymerization process with a set of experimental results.

The optical density accounts for inviable cells as well as viable cells, whereas the microbial population corresponds to viable cells. Figure 4 shows that the OD 630 outcomes after cultivation for four days and seven days are sum of conversions of microbial population and inviable cells.

\section{Acknowledgment}

The authors thank Ms. Y. Shimizu for her technical support. This work was supported by JSPS KAKENHI Grant Number 16K05276. 


\section{References}

[1] J. R. HAINES AND M. ALEXANDER, Microbial Degradation of Polyethylene Glycol, APPLIED MICROBIOLOGY, May 1975, p. 621625

[2] DARYL F. DWYER AND JAMES M. TIEDJE, Degradaton of Ethylene Glycol and Polyethylene Glycols by Methanogenic Consortia, APPLIED AND ENVIRONMENTAL MICROBIOLOGY, July 1983. p. 185-190.

[3] NURIA OBRADORS AND JUAN AGUILAR, Efficient Biodgradation of High-Molecular-Weight Polyethylene Glycols by Pure Cultures of Pseudomonas stutzeri, APPLIED AND ENVIRONMENTAL MICROBIOLOGY, Aug. 1991. p. 2383-2388.

[4] Masaji Watanabe, Fusako Kawai, Masaru Shibata, Shigeo Yokoyama, Yasuhiro Sudate, Shizue Hayashi, Analytical and computational techniques for exogenous depolymerization of xenobiotic polymers, Mathematical Biosciences 192 (2004) 19-37.

[5] M. Watanabe and F. Kawai, Effects of microbial population in degradation process of xenobiotic polymers, In P. Howlett, M. Nelson, and A. J. Roberts, editors, Proceedings of the 9th Biennial Engineering Mathematics and Applications Conference, EMAC-2009, vol 51 ANZIAM J., pp C682--C96, September 2010 http://journal.austms.org.au/ojs/index.php/ANZIAMJ/article/view/2433

[6] Masaji Watanabe, Fusako Kawai, Mathematical modelling and computational analysis of enzymatic degradation of xenobiotic polymers, Applied Mathematical Modelling 30 (2006) 1497-1514

[7] Masaji Watanabe, Fusako Kawai, Sadao Tsuboi, Shogo Nakatsu, Hitomi Ohara, Study on Enzymatic Hydrolysis of Polylactic Acid by Endogenous Depolymerization Model, Macromolecular Theory and Simulations 16 (2007) 619-626.

[8] Masaji Watanabe, Fusako Kawai, Simulation for microbial depolymerization processes of polyethylene glycol, Proceedings of the 2013 International Conference on Advances in Intelligent Systems in Bioinformatics, Editors: Dr. Ford Lumban Gaol, Dr. Benfino Soewito, Prof. Dr. Mohamed Bououdina, Prof. Dr. Mu-Song Chen, intel-13, Advances in Intelligent Systems Research, Atlantis Press, February 2014, 71 - 76. ISBN: 978-94-6252-000-4, ISSN: 1951-6851 http://www.atlantis-press.com/php/pub.php?publication=intel-13

[9] Masaji Watanabe, Fusako Kawai, Numerical Techniques for Inverse Problems from Modeling of Microbial Depolymerization Processes, International Journal of Applied Engineering Research ISSN 0973-4562 Volume 11, Number 8 (2016) pp 5461-5468. http://www.ripublication.com/ijaer16/ijaerv11n8_18.pdf

[10] Masaji WATANABE and Fusako KAWAI, Simulation of Microbial Depolymerization Process with Exponential Consumption of Carbon Source, 2016 International Conference on Computer, Mechatronics and Electronic Engineering (CMEE 2016), DEStech Publications, Inc., Lancaster, Pennsylvania, U.S.A., 2016, 426-431, ISBN: 978-1-60595406-6

[11] Masaji Watanabe, Fusako Kawai, Numerical Techniques for Simulation of Microbial Depolymerization Process with Time Factor of Degradation Rate, Submitted.

[12] M Watanabe and F Kawai, Computational analysis for biodegradation of exogenously depolymerizable polymer, 2017 the 5th International Conference on Mechanical Engineering, Material Science and Civil Engineering, December, 2017, Kuala Lumpur, Malaysia, 012015, IOP Conference Series: Materials Science and Engineering, Published online: 6 April 2018. IOP Publishing http://iopscience.iop.org/issue/1757$899 \mathrm{X} / 324 / 1$

[13] FUSAKO KAWAI AND SHOGO ENOKIBARA, Symbiotic Degradation of Polyethylene Glycol (PEG) 20,000-Phthalate Polyester by Phthalate Ester Ester- and PEG 20,000-Utilizing Bacteria, JOURNAL OF FERMENTATION AND BIOENGINEERING, Vol. 82, No.6, 575579, 1996.
[14] [14] MASAJI WATANABE, FUSAKO KAWAI, APPLICATION OF THE NEWTON'S METHOD AND BISECTION METHOD TO THREE PARAMETER PROBLEM FOR MICROBIAL DEPOLYMERIZATION PROCESS, Proceedings, ISER INTERNATIONAL CONFERENCE, 1st - 2nd April, 2018| Venue: Kuala Lumpur, Malaysia

[15] [15] M Watanabe and F Kawai, Computational study of bacterial depolymerization process of xenobiotic polymer, Submitted.

[16] Masaji Watanabe and Fusako Kawai, Numerical Study of Exogenous Type Microbial Depolymerization Process, 12th PATTAYA International Conference on Chemical, Agricultural, Biological and Environmental Sciences (PCABES-18), Pattaya (Thailand) May 31-June 2, 2018. ISBN: 978-93-86878-17-5

About Author (s):

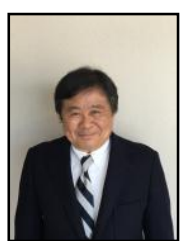

"Experimental outcomes obtained by cultivation of microbial consortium E-1 in culture media, in which PEG was the only carbon source, were incorporated in a numerical study. Results from a previous study were incorporated in a numerical simulation of an exogenous type depolymerization of PEG." 